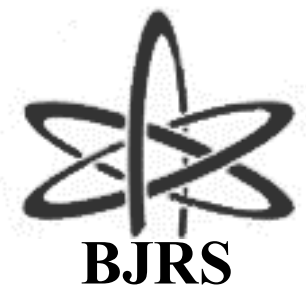

BRAZILIAN JOURNAL

$\mathrm{OF}$

RADIATION SCIENCES

08-01 (2020) 01-20

\title{
Riscos que comprometem a segurança do paciente no tratamento de teleterapia conformacional
}

\author{
Salvador ${ }^{a, b}$ C. S., Borges ${ }^{a}$ L. M., Dorow ${ }^{a}$ P. F., Felipe ${ }^{b}$ D. S., Rodrigues ${ }^{b}$ P. M., \\ Pereira $^{\mathrm{a}, \mathrm{b}}$ J. G, Pizzio ${ }^{\mathrm{b}}$ A. T \\ ${ }^{a}$ Instituto Federal de Educação, Ciência e Tecnologia de Santa Catarina, 88020-300, Av. Mauro Ramos, 950 - Centro, \\ Florianópolis/SC. \\ ${ }^{\mathrm{b}}$ Centro de Pesquisas Oncológicas, 88034-000, Rod. Admar Gonzaga, 655, SC 404 - Itacorubi, Florianópolis/SC.
} carolinesalvador09@gmail.com

\section{RESUMO}

O objetivo deste estudo foi analisar os riscos que comprometem a segurança do paciente no tratamento de teleterapia conformacional. Para isso, realizou-se uma pesquisa de campo, quali-quantitativa-exploratória, descritiva. Somente as etapas executadas por profissionais das técnicas radiológicas fizeram parte da análise de risco. Para a coleta de dados, utilizou-se observação assistemática com anotações em diário de campo e, diretamente nos quadros da ferramenta Análise do Modo e do Efeito de Falhas na Assistência à Saúde (HFMEA). Participaram dezesseis profissionais, quatro destes integraram a Equipe HFMEA, que realizou a aplicação da ferramenta. Os processos foram divididos em: 1- deslocamento; 2- primeiro dia de tratamento e; 3demais dias de tratamento. Os resultados constataram que o maior número de etapas e modos de falhas potenciais ocorre no primeiro dia de tratamento, e que a maioria dos modos de falhas potenciais dos três processos foi considerada tolerável e é relacionada, principalmente, a erros humanos. Na análise dos modos de falhas potenciais intoleráveis, constatou-se que em 59,37\% a intervenção era necessária, sendo propostas ações preventivas às falhas.

Palavras-chave: Análise do Modo e do Efeito de Falhas na Assistência à Saúde, Radioterapia, Proteção radiológica, Segurança do paciente, Análise de risco. 


\begin{abstract}
The objective of this study is to analyze the risks that compromise patient safety in the treatment of conformational teletherapy. To accomplish it a field research, qualitative-quantitative-exploratory, descriptive was conducted. Only the steps performed by professionals of the radiological techniques were part of the risk analysis. For data collection, non-systematic observation with field diary notes was used, and directly in the tables of the Healthcare Failure Mode and Effect Analysis (HFMEA) tool. Sixteen professionals participated, four of them were part of the HFMEA Team, which carried out the application of the tool. The processes were divided into: 1 -displacement; 2- the first day of treatment and; 3- other days of treatment. The results found that the greatest number of steps and potential failures modes occur on the first day of treatment and; that most of the potential failures modes of the three processes was considered tolerable and are mainly related to human errors. In the analysis of the intolerable potential failures modes, it was found that in $\mathbf{5 9 . 3 7 \%}$ the intervention was necessary and preventive actions were proposed.
\end{abstract}

Keywords: Healthcare Failure Mode and Effect Analysis, Radiotherapy, Radiation protection, Patient safety, Risk analysis. 


\section{INTRODUÇÃO}

De acordo com a Agência Internacional de Energia Atômica (IAEA), foram relatados por meio do seu Sistema de Comunicação e Aprendizagem de Segurança para Radioterapia (SAFRON), mundialmente 858 incidentes em teleterapia, no período de 1986 a outubro de 2019. Destes incidentes, 59,67\% atingiram o paciente [1]. No Brasil, até janeiro de 2015, foram relatados pela Comissão Nacional de Energia Nuclear (CNEN) quatro acidentes entre 2011 e 2014, entre eles, um com consequência fatal [2]. Infelizmente, no país ainda não há a cultura de relatar os incidentes ocorridos. Os que foram divulgados são aqueles que tiveram repercussão na imprensa. Isso não significa que não haja acidente e sim falta de divulgação [3].

No início do século XXI, a segurança do paciente tornou-se uma das principais preocupações mundiais, constituindo um tema de relevância crescente entre pesquisadores do todo o mundo [4].

Garantir segurança e qualidade no processo de radioterapia exige que uma série de procedimentos sejam realizados adequadamente [5]. Existe a necessidade de métodos prospectivos, estruturados e sistêmicos para identificar as fraquezas dos procedimentos realizados e para se antecipar às falhas potenciais, incluindo a avaliação e a comparação de riscos potenciais para cada falha potencial identificada. O que permite a seleção das ações a serem realizadas para um maior benefício do paciente [6].

De acordo com a Organização Mundial da Saúde, é evidente na literatura que os incidentes de radioterapia estão principalmente relacionados a erros humanos [7] e 24\% das publicações mais recentes consideram o fator humano na gestão e na minimização de erros em radioterapia [8]. Dessa forma, pesquisas relacionadas à segurança do paciente em radioterapia são essenciais, levando-se em consideração o expressivo número de incidentes ocorridos em radioterapia, a gravidade com que podem atingir os pacientes e a necessidade de contribuir com a prevenção de erros humanos.

Diante disso, o presente estudo teve como objetivo analisar os riscos que comprometem a segurança do paciente no tratamento de teleterapia conformacional. 


\section{MATERIAIS E MÉTODOS}

Trata-se de uma pesquisa de campo, quali-quantitativa-exploratória, descritiva. Utilizou-se para análise dos riscos a ferramenta HFMEA $^{\mathrm{TM}}$ - Análises do Modo e do Efeito de Falhas na Assistência à Saúde -, desenvolvida em 2002 por pesquisadores do Centro Nacional de Segurança de Pacientes, do Departamento de Assuntos de Veteranos dos Estados Unidos (VA).

Essa ferramenta é um modelo de análise prospectiva híbrida que combina conceitos encontrados em Análises do Modo e do Efeito de Falhas (Failure Mode and Effects Analysis - FMEA) e Análises de Perigos e Ponto Crítico de Controle (Hazard Analysis and Critical Control Point HACCP), com ferramentas e definições encontradas na ferramenta Análise de Causa Raiz (Root Cause Analysis - RCA) do VA. Sua metodologia é desenvolvida em cinco etapas: definir um escopo; montar uma equipe; descrever graficamente o processo; realizar a análise de perigos; e propor ações e medidas de resultados [9].

Justifica-se a escolha dessa ferramenta, pois é considerada útil para descrever a confiabilidade do sistema, comparar modelos alternativos e orientar o processo de melhoria [10]. Cabe salientar que, tanto no Brasil quanto no exterior, existem poucos estudos relacionados à aplicação da ferramenta HFMEA $^{\mathrm{TM}}$ na teleterapia.

O local escolhido para o desenvolvimento desta pesquisa foi o setor de radioterapia de um centro de tratamento oncológico, localizado no sul do Brasil, onde técnicos e tecnólogos em radiologia, denominados profissionais das técnicas (PTR's), executam diariamente mais de 120 procedimentos teleterápicos. Entre as diferentes técnicas de teleterapia, optou-se pela teleterapia conformacional, já que esta é a técnica de tratamento mais utilizada no local da realização da pesquisa. O local possui duas salas de tratamento de teleterapia com dois aceleradores lineares, Clinac 2100SC e Clinac 600C/D, ambos da marca Varian Medical System ${ }^{\circledR}$, o serviço conta com o sistema de registro e verificação (RV) Aria ${ }^{\circledR}$.

Participaram da pesquisa 16 profissionais integrantes da equipe multidisciplinar que atua no setor de radioterapia, sendo eles: médico rádio-oncologista, físicos médicos e profissionais das técnicas radiológicas - PTR's (técnicos e tecnólogos em radiologia). Destes 16 profissionais, foram eleitos quatro experts da área de teleterapia para compor a equipe que aplicou a ferramenta, denominada Equipe HFMEA, os quatro participantes da equipe foram escolhidos por serem 
oriundos de diferentes especialidades, por possuírem ou estarem cursando pós-graduação e pela compatibilidade de horários para participação nas reuniões da pesquisa. Os PTR's atuantes (12) foram observados assistematicamente para coleta de dados.

A ferramenta HFMEA é uma metodologia de análise de riscos desenvolvida para aplicação na área de assistência à saúde, podendo ser adaptada e aplicada em áreas específicas dentro deste contexto. Portanto, para utilização nesta pesquisa a ferramenta foi adaptada, segundo ajuste sugerido pela Equipe HFMEA, no passo “propor ações e medidas de resultados”. Essa adaptação foi validada, anteriormente à sua aplicação, por intermédio de consenso, o qual ocorreu em reuniões da referida equipe.

O desenvolvimento da pesquisa aconteceu entre dezembro de 2017 e julho de 2018. A Equipe HFMEA aplicou a metodologia nos seguintes passos consecutivos: (1) escolha da área a ser analisada; (2) escolha da equipe multidisciplinar; (3) identificação das etapas, dos Modos de Falhas Potenciais - MFP - e das causas potenciais das falhas; (4) avaliação dos riscos dos MFP e; (5) identificação de recomendações e propostas de ações de prevenção aos MFP.

(1) A análise concentrou-se nos processos realizados pelos PTR's (técnicos e tecnólogos em radiologia) nas salas de tratamento de teleterapia conformacional, estendendo-se desde o procedimento de deslocamento (deslocamento da marcação inicial do isocentro de tratamento, realizada durante a simulação na tomografia computadoriza, para o local de tratamento definido pelo rádio-oncologista), passando pelo primeiro dia de tratamento até a alta do tratamento. Apenas as etapas relacionadas ao tratamento de teleterapia conformacional fizeram parte da análise de risco, deste modo, as técnicas de radioterapia estereotáxica, radiocirurgia, radioterapia de intensidade modulada e terapia com elétrons não integraram a pesquisa.

(2) A pesquisa contou com a colaboração de 16 profissionais, sendo um médico rádio-oncologista, dois físicos médicos e treze profissionais das técnicas radiológicas (PTR's - técnicos e tecnólogos em radiologia).

(3) A autora/pesquisadora, tecnóloga em radiologia, que atua como PTR no local da pesquisa, realizou a identificação dos processos de forma direta a partir da observação assistemática, sendo estes mapeados em diário de campo. A observação de forma assistemática permitiu o contato direto com os profissionais em seu ambiente natural de trabalho, sem participação nem interação, observando seus comportamentos verbais e não verbais, não havendo interferência da pesquisadora, ou seja, a 
observação assistemática não causou desvios na coleta de dados. Posteriormente a identificação dos nove processos, selecionou-se aqueles executados por PTR's nas salas de tratamento e por meio de observação assistemática dos PTR's participantes, identificou-se e descreveu-se detalhadamente as etapas dos processos e as ações realizadas por estes em cada etapa. Em seguida, foram transcritas e enumeradas para quadros HFMEA ${ }^{\mathrm{Tm}}$ distintos. A partir deste momento, a pesquisa contou com a colaboração da Equipe HFMEA, que por intermédio de reuniões realizadas no próprio setor, validava e acrescentava informações à ferramenta. A equipe identificou e enumerou os MFP para cada uma das etapas, na sequência identificou as causas potenciais desses MFP.

É importante destacar que, apesar da aplicação da ferramenta ser focada nas ações executadas por PTR's, todos os MFP e as suas causas potenciais foram listadas, independentemente da origem. No entanto, somente nos MFP e nas causas potencias resultantes de ações realizadas por PTR's realizou-se as pontuações de severidade e de probabilidade. Logo, os demais MFP e as causas potenciais não foram analisados e/ou categorizados, por serem de responsabilidade institucional ou de outros profissionais.

(4) Em relação à avaliação dos riscos, foram realizadas as ponderações como base nas definições e pontuações publicadas conforme abaixo [9].

A probabilidade é uma medida da probabilidade de ocorrência de falhas e suas classificações incluem: (a) Frequente: provavelmente acontece imediatamente ou dentro de um curto período (várias vezes em um ano); (b) Ocasional: possivelmente vai ocorrer (várias vezes entre um a dois anos); (c) Incomum: possível ocorrer (em algum momento entre dois a cinco anos); (d) Remoto: não é provável que ocorra (em algum momento entre cinco a 30 anos).

A Severidade é uma medida do potencial efeito sobre o modo de falha, sendo dividida nas categorias: (a) Catastrófica: morte ou perda permanente importante de função (sensorial, motor, fisiológica ou intelectual), procedimento sobre o paciente errado ou parte do corpo errado; (b) Crítica: diminuição permanente do funcionamento corporal (sensorial, motor, fisiológico ou intelectual), desfiguração, intervenção cirúrgica necessária, aumento do nível de cuidados para 3 ou mais pacientes; (d) Moderada: aumento do nível de cuidados para 1 ou 2 pacientes; (e) Desprezível: sem lesões, nem aumento do nível de cuidados.

Seguidamente, encontra-se o produto das pontuações para cada MFP e concomitantemente sua classificação, conforme Matriz de Pontuação de Perigo (MPP) apresentada na Tabela 1. 
Tabela 1: Matriz de Pontuação de Perigo (MPP)

\begin{tabular}{|c|c|c|c|c|c|}
\hline \multicolumn{2}{|c|}{} & \multicolumn{4}{c|}{ Severidade do efeito } \\
\cline { 2 - 7 } & $\begin{array}{c}\text { Xatastrófica } \\
\text { (peso 4) }\end{array}$ & $\begin{array}{c}\text { Crítica } \\
\text { (peso 3) }\end{array}$ & $\begin{array}{c}\text { Moderada } \\
\text { (peso 2) }\end{array}$ & $\begin{array}{c}\text { Desprezível } \\
\text { (peso 1) }\end{array}$ \\
\hline \multirow{4}{*}{ Probabilidade } & Frequente (peso 4) & 16 & 12 & 8 & 4 \\
\cline { 2 - 7 } & Ocasional (peso 3) & 12 & 9 & 6 & 3 \\
\cline { 2 - 7 } & Incomum (peso 2) & 8 & 6 & 4 & 2 \\
\cline { 2 - 7 } & Remoto (peso 1) & 4 & 3 & 2 & 1 \\
\hline
\end{tabular}

Fonte: Adaptado de DeRosier et al. (2002, p. 267).

Considerando as definições e instruções de DeRosier [9], realizou-se uma análise aprofundada para cada MFP intolerável isoladamente por meio da Árvore de Decisão (Figura 1) quanto a necessidade de novas ações com base: na falta de detectabilidade (a probabilidade de detecção da falha ou seu efeito antes que ocorra), na criticalidade (mede se o sistema inteiro falhará se esta parte do processo falhar) e na ausência de medidas de controle efetivas (medida que elimina ou reduz significativamente a probabilidade de a falha ocorrer).

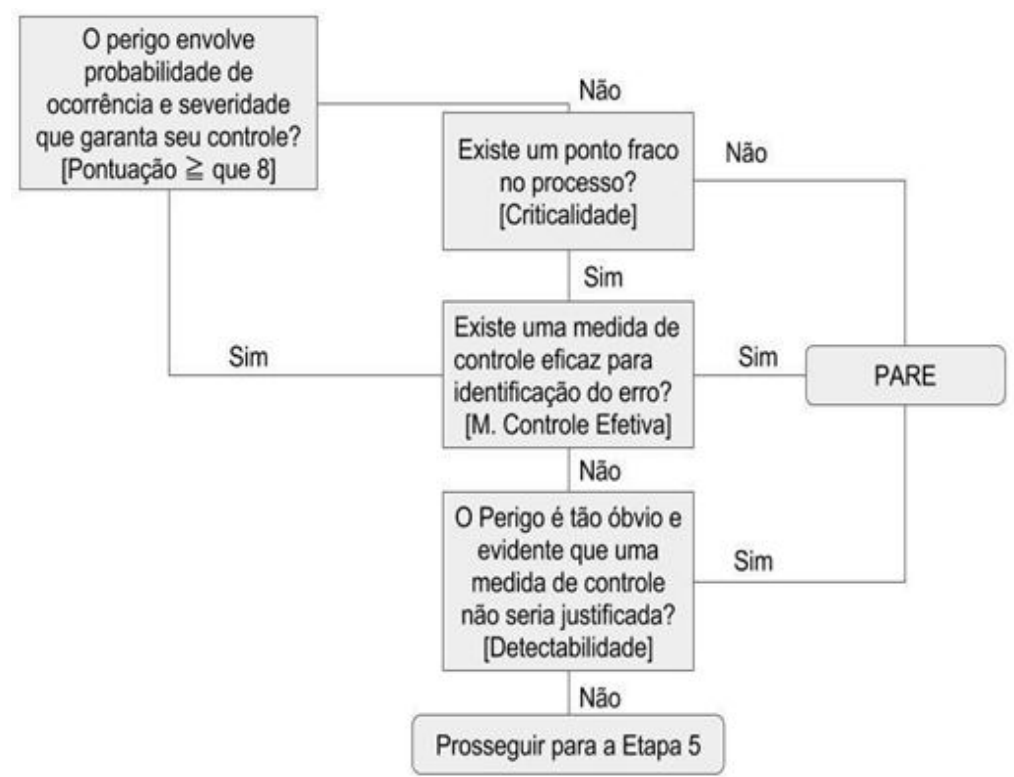

Figura 1: Árvore de Decisão

Fonte: Adaptado de DeRosier et al. (2002, p. 255). 
(5) Com base na avaliação da Árvore de Decisão, a equipe identificou as prioridades de intervenção definindo que tipo de ação deve-se realizar para cada MFP (controlar, aceitar ou eliminar), ou justificando o motivo para parar a análise. Quando a ação definida pela equipe foi controlar ou eliminar o MFP, identificou-se em organizações reconhecidas nacionais e internacionais, como Comissão Nacional de Energia Nuclear - CNEN [11] - e Comissão Internacional de Proteção Radiológica - ICRP [6] -, recomendações para prevenir determinado MFP, além de propor ações preventivas para o MFP, nas quais nenhuma recomendação foi encontrada. Na última coluna da ferramenta, detectou-se pessoas responsáveis para realizar/assegurar a realização de cada ação.

O estudo foi aprovado pelo Comitê de Ética em Pesquisa com seres humanos, parecer 2.375.568.

\section{RESULTADOS}

Os processos envolvidos na realização do tratamento teleterápico conformacional, sem intercorrências, são demonstradas no fluxograma (Figura 2).

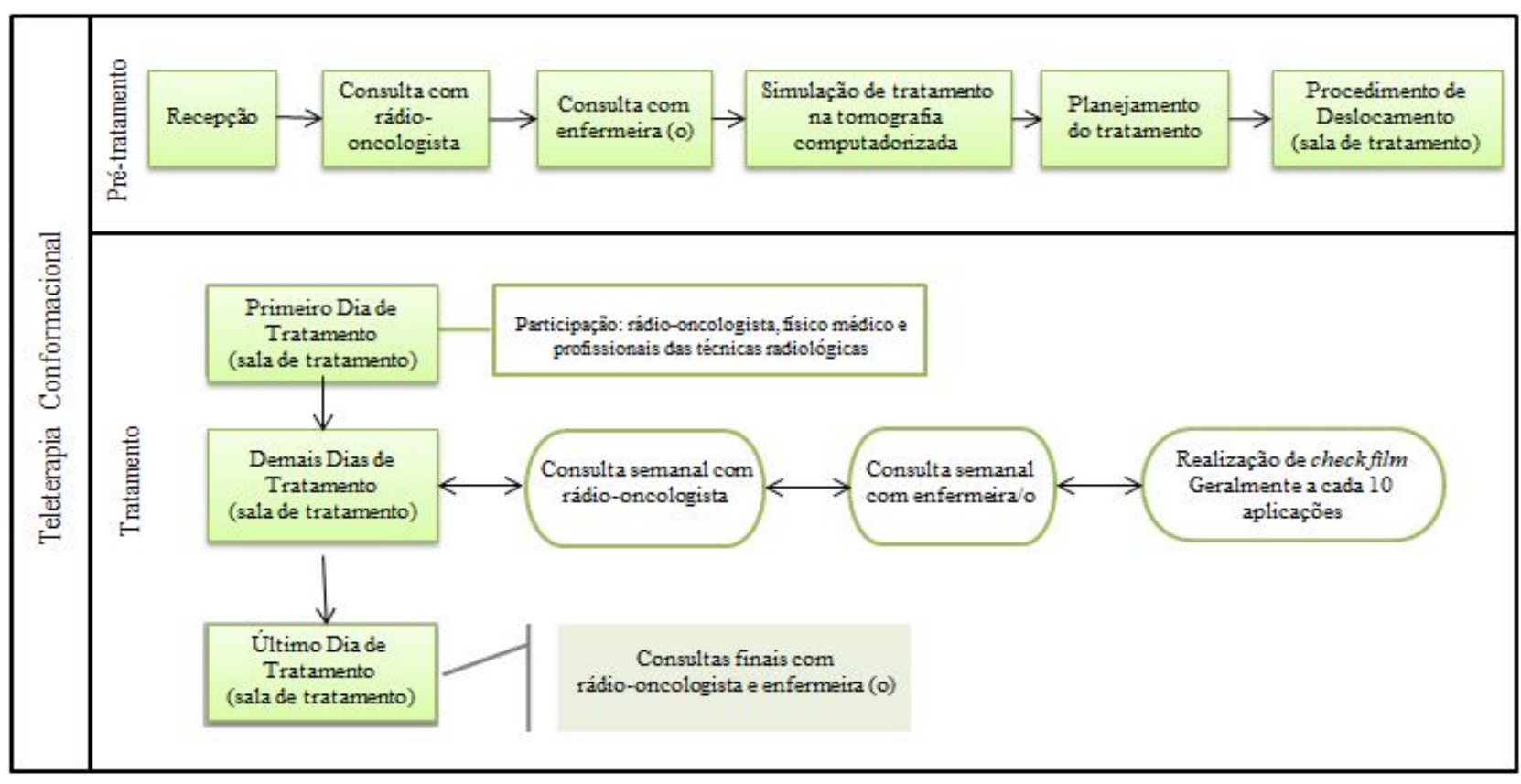

Figura 2: Processos envolvidos no tratamento de teleterapia conformacional 
É importante destacar que a Figura 2 demonstra os processos sem intercorrências, permitindo assim a visualização de maneira clara e objetiva de todo o processo desenvolvido para a realização de um tratamento de teleterapia. E que, apesar de não estarem identificados na figura, todos os MFP e as causas potenciais das ações realizadas por PTR's foram objeto de análise de risco.

Como pode ser observado na Figura 2, são realizados nove processos para o tratamento de teleterapia conformacional, no setor de radioterapia do local pesquisado, estes processos são realizados por diversos profissionais, sendo que o primeiro dia de tratamento envolve uma equipe multidisciplinar composta por médico rádio-oncologista, físico médico e profissionais das técnicas radiológicas, em conformidade com a legislação da Comissão Nacional de Energia Nuclear [11].

A aplicação da ferramenta HFMEA $^{\mathrm{TM}}$ foi realizada somente nos processos executados por PTR's nas salas de tratamento teleterápico, sendo eles: (1) Deslocamento; (2) Primeiro dia de tratamento e; (3) Demais dias de tratamento (até a alta do paciente), cada processo foi analisado em um quadro HFMEA, separadamente.

Em todos os quadros HFMEA, listou-se as etapas, os modos de falhas potenciais (MFP) para cada uma delas e as causas potenciais das falhas. É importante relembrar que, apesar da aplicação da ferramenta ser focada nas ações executadas por PTR's, listou-se todos os MFP e as suas causas potenciais, independentemente da origem.

Apesar da pesquisa contemplar três processos de um modo amplo, listando todos os MFP e as causas potenciais envolvidas, somente foram realizadas as pontuações de severidade e de probabilidade nos MFP resultantes de ações realizadas por PTR's, ou seja, os demais não fizeram parte da análise por serem de responsabilidade institucional ou de outros profissionais. Com o mesmo critério, listou-se todas as causas potenciais dos MFP, dando assim uma maior abrangência à pesquisa. Dessa forma, os MFP e as causas potenciais de responsabilidade institucional ou de outros profissionais não foram analisados, tampouco categorizados.

De acordo com o resultado do produto das pontuações severidade e probabilidade, na Matriz de Pontuação de Perigo (Quadro 1), classificou-se o MFP em: aceitável, tolerável ou intolerável. Os MFP intoleráveis passaram pela análise da Árvore de Decisão, seguindo para o próximo passo ou justificando a razão para parar a análise. Descreveu-se ações preventivas e pessoas responsáveis por elas em todos MFP onde a Equipe HFMEA julgou necessário controlar ou eliminar o MFP, identificando e melhorando as medidas de prevenção e controle já existentes no processo, assim 
como novas e recomendadas por organizações reconhecidas nacionais e internacionais. A seguir, o detalhamento de cada um dos três processos analisados.

\subsection{Processo de deslocamento tem menor índice de modos de falhas intoleráveis}

Dos três processos analisados, o processo de deslocamento apresentou a menor porcentagem de modos de falhas potenciais intoleráveis e a maior porcentagem de modos de falhas potenciais toleráveis. Listou-se neste processo 15 etapas desenvolvidas por PTR's, desde a seleção do paciente no Sistema Aria $^{\circledR}$ até o arquivamento dos check films realizados na ficha técnica e o seu encaminhamento para análise do rádio-oncologista.

Nessas etapas, listou-se 63 modos de falhas potenciais com 39 tipos de causas, apontados 278 vezes.

Em se tratando dos modos de falhas potenciais, pontuou-se 92,06\% (severidade e probabilidade) e 7,93\% não foram pontuados, por não serem falhas resultantes de ações realizadas por PTR's.

Em relação aos MFP pontuados na MPP, 3,44\% correspondem a intoleráveis (MPP maior ou igual a 8); 94,82\% a toleráveis (MPP 6 e 4); e 1,72\% a aceitáveis (MPP menor ou igual a 3).

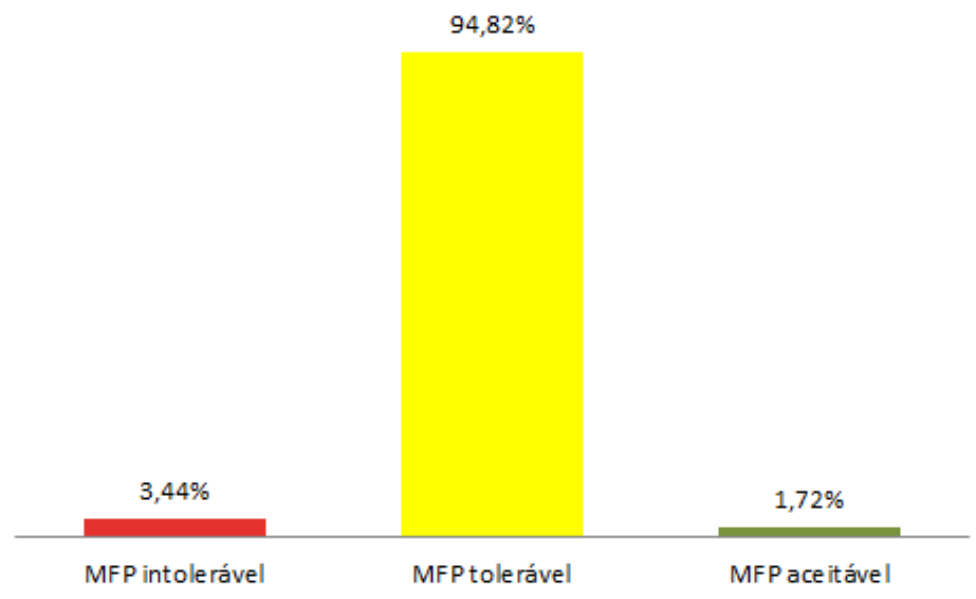

Figura 3: MFP no processo de deslocamento

Ao se analisar os tipos de causas, em 33,33\% das vezes considerou-se de responsabilidade dos PTR's e 66,66\% de responsabilidade institucional ou de outros profissionais. Entre os tipos de causas de responsabilidade dos PTR's, $61,53 \%$ correspondem a causas de uma única falha e 38,46\% associadas a mais de uma falha. E entre os tipos de causas de responsabilidade institucional ou de 
outros profissionais $73,07 \%$ correspondem a causas de uma única falha e 26,92\% associadas a mais de uma falha.

Em $64,74 \%$ das vezes que as causas foram apontadas considerou-se de responsabilidade dos PTR's, em 35,25\% como sendo de responsabilidade institucional ou de outros profissionais.

\subsection{Legislação aumenta barreiras contra falhas potenciais no primeiro dia de tratamento de teleterapia}

$\mathrm{O}$ processo de primeiro dia de tratamento apresentou o maior número de etapas listadas somando 25 etapas desenvolvidas pelos PTR's, desde a seleção do paciente no Sistema Aria ${ }^{\circledR}$ até o arquivamento ou encaminhamento da ficha técnica aos setores responsáveis.

Nessas etapas, também foi elencado o maior número de modos de falhas potenciais, 127. Nestes, 47 tipos de causas potenciais, apontados 660 vezes.

Entre os MFP, pontuou-se 92,91\% e 7,08\% não foram pontuados, por não serem falhas resultantes de ações realizadas por profissionais das técnicas radiológicas.

Em relação aos MFP pontuados na MPP, 12,71\% correspondem a intoleráveis (MPP maior ou igual a 8); 72,03\% a toleráveis (MPP 6 e 4); e 15,25\% a aceitáveis (MPP menor ou igual a 3).

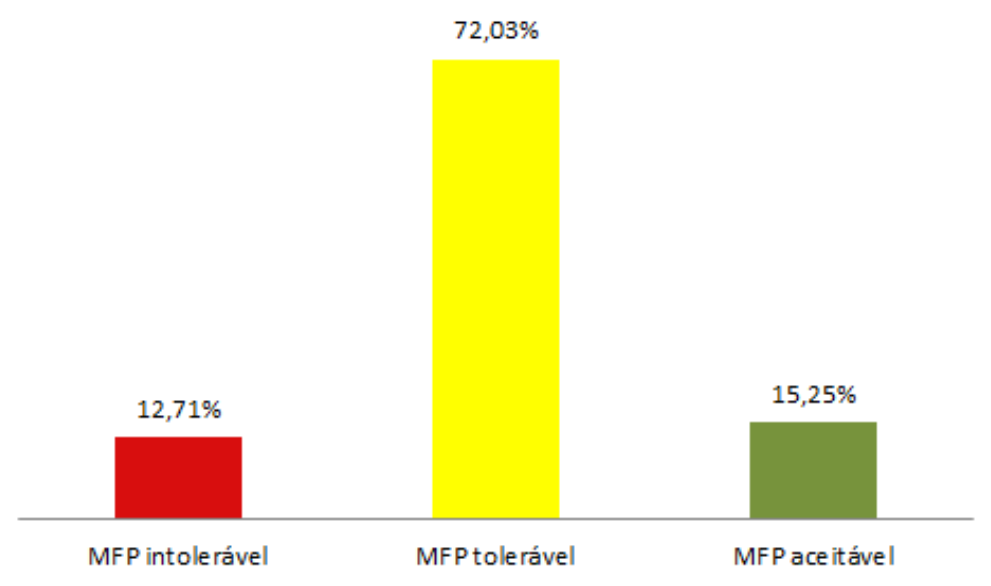

Figura 4: MFP no processo de primeiro dia de tratamento 
Ao se analisar os tipos de causas, consideraram-se $31,25 \%$ como sendo de responsabilidade dos PTR's e 68,75\% de responsabilidade institucional ou de outros profissionais. Entre os tipos de causas de responsabilidade dos PTR's, 53,25\% correspondem a causas de uma única falha e 46,66\% associadas a mais de uma falha. Já entre os tipos de causas de responsabilidade institucional ou de outros profissionais, $66,66 \%$ correspondem a causas de uma única falha e 33,33\% são associadas a mais de uma falha.

Em $63,18 \%$ das vezes que as causas foram apontadas, considerou-se de responsabilidade dos PTR's, em 36,81\% como sendo de responsabilidade institucional ou de outros profissionais.

\subsection{Maior índice de modos de falhas potenciais intoleráveis}

No processo de demais dias de tratamento, listou-se 22 etapas desenvolvidas por profissionais das técnicas radiológicas, desde a seleção do paciente no Sistema Aria ${ }^{\circledR}$ até o arquivamento ou encaminhamento da ficha técnica aos setores responsáveis.

Nessas etapas, listou-se 111 modos de falhas potenciais com 47 tipos de causas potenciais, apontados 550 vezes.

Entre os modos de falhas potenciais, pontuou-se $95,59 \%$ e 5,40\% não foram pontuados, por não serem falhas resultantes de ações realizadas por profissionais das técnicas radiológicas.

Em relação aos MFP pontuados na MPP, 14,28\% correspondem a intoleráveis (MPP maior ou igual a 8); 73,33\% a toleráveis (MPP 6 e 4); e 12,38\% a aceitáveis (MPP menor ou igual a 3).

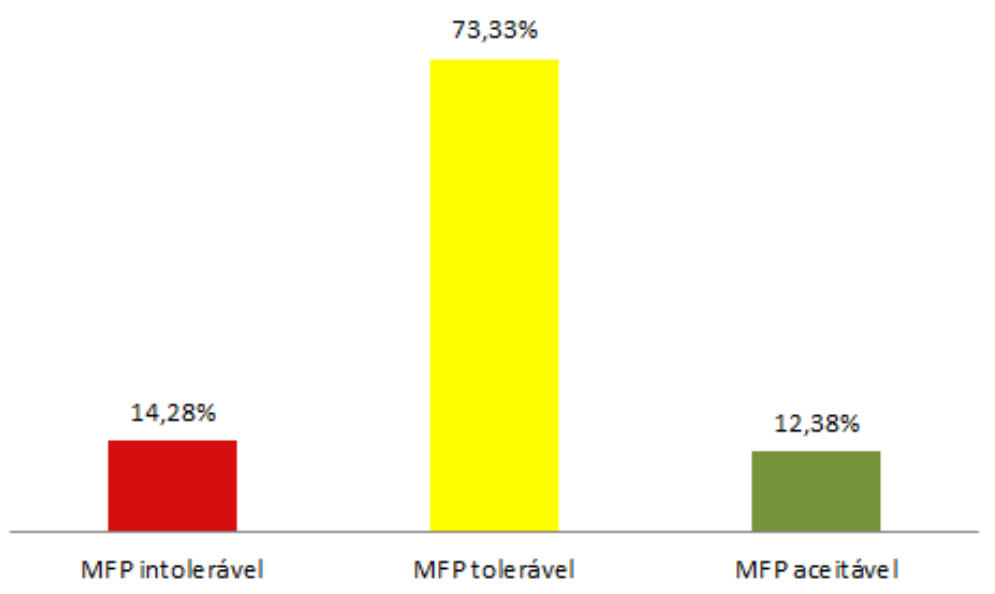

Figura 5: MFP no processo de demais dias de tratamento 
Ao se analisar os tipos de causas, considerou-se 34,04\% como sendo de responsabilidade dos PTR's e 65,95\% de responsabilidade institucional ou de outros profissionais. Entre os tipos de causas de responsabilidade dos PTR's, 56,25\% correspondem a causas de uma única falha e 43,75\% associadas a mais de uma falha. Já entre os tipos de causas de responsabilidade institucional ou de outros profissionais, $67,74 \%$ correspondem a causas de uma única falha e $32,25 \%$ associadas a mais de uma falha.

Em 63,63\% das vezes que as causas foram apontadas, considerou-se como sendo de responsabilidade dos PTR's, e em 36,36\% como sendo de responsabilidade institucional ou de outros profissionais. Estes dados comprovam que o trabalho envolvido na teleterapia é de fato multiprofissional.

Os principais tipos de causas considerados de responsabilidade dos PTR's nos três processos analisados (apontados quatro vezes ou mais) são: desatenção, negligência profissional, falta de tempo, falha na comunicação e falta de conhecimento, respectivamente. E os principais tipos de causas considerados de responsabilidade institucional ou de outros profissionais nos três processos (apontados quatro vezes ou mais) são: falta de treinamento/capacitação, alto volume de pacientes, ausência de protocolos institucionalizados, número insuficiente de profissionais, falha no equipamento, ambiente de trabalho inadequado, falta de manutenção, informação especificada incorretamente, não especificada na ficha técnica e; especificada incorretamente na ficha técnica, consecutivamente.

\section{DISCUSSÃo}

Conforme relatado pela International Commission on Radiological Protection, os profissionais das técnicas radiológicas (PTR's) têm participação em $20 \%$ dos casos de erro [6]. Como esta pesquisa utiliza uma ferramenta prospectiva, avaliou-se modos de falhas potenciais, ou seja, falhas possíveis de aconteceram nas ações realizadas por estes profissionais, por este motivo a porcentagem encontrada é maior. Os PTR's estão intimamente envolvidos no processo radioterapêutico, posto que o contato com o paciente é praticamente diário e estes executam o 
tratamento de forma direta na entrega da dose, logo, os profissionais têm papel determinante na eficácia do tratamento.

A prevenção de erros é extremamente relevante em todos os procedimentos envolvidos no processo de tratamento de radioterapia. A publicação "Bonn call for action" traz como uma das dez ações para a melhoria da proteção radiológica em medicina na próxima década: "melhorar a prevenção de incidentes e acidentes com radiação utilizada em contexto clínico", sendo uma das subações implementar métodos prospectivos de análise dos riscos para reforçar a segurança na prática clínica [12].

Há menção em várias publicações de melhorias na qualidade dos serviços, em diversas áreas da saúde após a aplicação da ferramenta HFMEA ${ }^{\text {TM }}$ [13]. Observa-se que os processos se tornam mais confiáveis a partir de metodologias de análise de risco, a abordagem sistemática da HFMEA ${ }^{\mathrm{TM}}$ é considerada de fácil aplicabilidade nos processos de saúde [9], mostrando-se útil [14-16] e gerando conhecimento profundo do processo radioterapêutico analisado, apesar de ainda ser pouco utilizada nessa área [17].

Analisou-se na presente pesquisa três procedimentos do processo de teleterapia conformacional: deslocamento, primeiro dia de tratamento e demais dias de tratamento. Foram encontradas 62 etapas, 301 modos de falhas potenciais e 58 tipos de causas, excluindo-se os tipos de causas que se repetiam nos modos de falha potenciais dos três procedimentos analisados. Apontou-se 1488 vezes tais tipos de causas nos MFP analisados.

O ambiente de trabalho envolvido nos processos radioterápicos apresenta diversos fatores de risco que expõem o trabalhador à possibilidade do erro, agravados pela complexidade do processo multitarefas e por falhas de usabilidade do sistema de tratamento [8]. Dado o fato que falhas humanas são inerentes a qualquer processo, ações de melhoria devem ser focadas na prevenção de erros e melhoria dos processos, consequentemente, na segurança dos pacientes.

Com base nos resultados obtidos na aplicação da ferramenta HFMEA, constatou-se que o maior número de etapas e MFP ocorrem no primeiro dia de tratamento, o fato demanda maior atenção dos profissionais, posto que um erro nesta etapa do processo pode prejudicar a reprodutibilidade do tratamento. 
Corroborando com o tema, a pesquisa de Dorow et al. [18] indica a importância da comunicação no processo que envolve o trabalho interdisciplinar na radioterapia para garantir a reprodutibilidade do tratamento.

Apesar de serem listados mais tipos de causas considerados de responsabilidade institucional ou de outros profissionais, houve uma maior frequência de causas resultantes de ações dos PTR's nos três processos.

Constatou-se que, dos MFP, 281 foram pontuados por serem resultantes de ações realizadas por PTR's, sendo que 11,38\% receberam pontuação respectiva à MFP aceitável; 77,22\% à tolerável e; $11,38 \%$ à intolerável.

Os MFP intoleráveis passaram pela análise da Árvore de Decisão (Figura 1), levando em consideração a criticalidade, a ausência de medidas de controle efetivas e a falta de detectabilidade, justificando-se o motivo para parar a análise ou indicando uma ação: aceitar, controlar ou eliminar determinado MFP, a Equipe HFMEA optou em todos os casos, quando a ação era necessária, por controlar o MFP, já que, por consenso, não há como eliminar falhas relacionadas ao erro humano, pois os profissionais são falíveis.

Com a análise dos MFP na Árvore de Decisão, identificou-se as prioridades de intervenção, considerou-se que em 59,37\% dos MFP a intervenção era necessária e em 40,62\%, não era necessária, nestes, justificou-se o motivo para parar a análise e, naqueles, propôs-se ações preventivas aos MFP.

Com relação às recomendações encontradas em organizações reconhecidas nacionalmente, têmse, por exemplo, que o titular do serviço de radioterapia deve garantir que haja, no mínimo, dois técnicos em radioterapia por turno, por equipamento; adicionalmente, deve disponibilizar os recursos necessários para: realizar treinamento anual de indivíduos ocupacionalmente expostos tanto para atuação em situações normais de trabalho, quanto em situações de incidente ou acidente; minimizar a probabilidade de ocorrência de acidentes [11]. Quanto às recomendações encontradas em organizações reconhecidas internacionalmente, têm-se, por exemplo, que é essencial que todo tratamento seja cuidadosamente documentado. As radiografias e todos os documentos do paciente devem ser claramente identificados e datados para evitar confusão entre pacientes ou entre diferentes estágios do tratamento de um mesmo paciente. Se ocorrer um acidente, esses documentos 
serão essenciais para o rádio-oncologista em qualquer decisão relativa a cuidados adicionais do paciente [6].

Atribui-se o fato de o número de MFP intoleráveis com necessidade de intervenção não ser mais expressivo à utilização no local pesquisado de um sistema de registro e verificação (RV), funcionando como medida de controle efetiva para a identificação e barreira das falhas.

Este tipo de sistema melhora a segurança dos tratamentos, evitando que um tratamento seja executado a menos que todas as informações originais do planejamento do tratamento, como ângulo do gantry, do colimador, formato do campo, coincidam com as informações da aplicação [19].

Na percepção da Equipe HFMEA, os MFP toleráveis se sobrepõem aos aceitáveis e intoleráveis. A baixa porcentagem atribuída aos MFP aceitáveis resulta do fato de existir risco à segurança do paciente nos procedimentos analisados. Dessa forma, somente os MFP com baixíssima probabilidade ou que resultam em nenhum dano ao paciente foram classificados como aceitáveis.

A baixa porcentagem de MFP intoleráveis no processo de deslocamento é consequente do fato de que, mesmo que fosse atribuída uma alta pontuação para a probabilidade, a pontuação atribuída à severidade foi baixa em praticamente todos os casos. Logo, o produto das pontuações resulta em MFP classificados como toleráveis ou aceitáveis, dependendo das pontuações atribuídas a cada MFP. Por consenso, decidiu-se que, mesmo que as falhas ocorram no processo de deslocamento, não geram dano ao paciente e/ou são barradas no processo de primeiro dia de tratamento, visto que, em conformidade com a legislação vigente, há a presença e o acompanhamento do rádiooncologista e do físico médico na entrega do primeiro dia de tratamento. Como destacado na pesquisa de Santo et al. [20], a presença dos dois profissionais no primeiro dia de tratamento é uma das barreiras mais importantes para a prevenção de acidentes.

$\mathrm{Na}$ análise de riscos realizada com a aplicação da ferramenta, a principal vantagem identificada está relacionada à multidisciplinaridade da Equipe HFMEA, o que gerou debates focados nas práticas diárias, relacionados aos possíveis modos de falhas, à cultura de segurança e à responsabilidade dos profissionais na otimização da segurança do paciente. O que possibilitou a melhoria do trabalho em equipe, a compreensão compartilhada das falhas potenciais envolvidas nos processos analisados.

A equipe também identificou limitações relacionadas à prática da mesma, como: a subjetividade na classificação de severidade e probabilidade; a necessidade de reunir um número considerável de 
pessoas por um longo período de tempo; a realização das pontuações, levando-se em consideração apenas os MFP e não as suas causas.

Desse modo, propõe-se como melhorias e sugestões para pesquisas futuras: adaptar as classificações de severidade e probabilidade conforme a realidade de cada área a ser analisada (na teleterapia, por exemplo, adaptar a severidade, categoria crítica para suspensão do tratamento por período necessário à recuperação do paciente); realizar as pontuações das causas potenciais em vez dos MFP's somente; alterar o valor dos pesos dados na Matriz de Pontuação de Perigo para possibilitar a determinação das pontuações sobre o ponto de vista individual, ou realizar essas pontuações somente com a Equipe reunida.

\section{CONCLUSÕES}

O presente estudo possibilitou analisar os riscos que comprometem a segurança do paciente no tratamento de teleterapia conformacional. Conclui-se que o profissional das técnicas radiológicas tem responsabilidade em várias etapas no processo envolvido no tratamento teleterápico sendo, portanto, fundamental na correta execução daquilo que é planejado, visando à reprodutibilidade do tratamento e à minimização de possíveis falhas na busca pela otimização da segurança do paciente oncológico.

A aplicação da ferramenta de análise de riscos HFMEA, mesmo complexa, mostrou-se útil na identificação de possíveis falhas que podem comprometer a segurança do paciente nas ações realizadas por profissionais das técnicas radiológicas em teleterapia conformacional. De forma geral, a ferramenta apresentou boa aplicabilidade, a principal vantagem é o fato de a Equipe ser multidisciplinar e a principal limitação é a necessidade de reunir muitas pessoas por um longo período de tempo. Seu sucesso depende diretamente da equipe envolvida, dos conhecimentos dos experts pertencentes à equipe acerca do processo analisado, das suas experiências, assim como do tempo disponível para aplicação da ferramenta. Dessa forma, torna-se necessário o apoio da gerência/gestor da instituição, tanto para disponibilizar o tempo essencial à aplicação, quanto para a implementação das recomendações. 
Foram identificadas e recomendadas ações de prevenção aos MFP de acordo com a HFMEA obtida para a instituição onde ela foi aplicada. Demais instituições devem verificar o risco envolvido em suas atividades de acordo com o fluxo do seu processo e das suas particularidades.

As ações preventivas recomendadas nesta pesquisa estão relacionadas principalmente ao desenvolvimento de protocolos e checklists e à realização de treinamento constante da equipe envolvida. Dessa maneira, torna-se necessário o apoio e o envolvimento real dos profissionais gestores.

A metodologia HFMEA é dinâmica, logo, recomenda-se a alimentação da ferramenta sempre que houver mudanças, como novos processos e/ou novas tecnologias que venham a ser implementadas na instituição. Adicionalmente, recomenda-se que, após a execução das recomendações de prevenção aos MFP seja realizada a reavaliação da HFMEA, com objetivo de atualizar as necessidades para os procedimentos analisados.

Sugere-se, ainda, a realização de novas pesquisas, aplicando a ferramenta de análise de riscos HFMEA, a partir das melhorias propostas neste trabalho, nas demais etapas do processo envolvido no tratamento de teleterapia, assim como em outros procedimentos realizados em departamentos de radioterapia.

\section{AGRADECIMENTOS}

Agradecemos ao local que permitiu a realização desta pesquisa, aos participantes, em especial aos membros da Equipe HFMEA, pela dedicação e pelo comprometimento com a melhoria dos serviços prestados. Especialmente, ao Instituto Federal de Educação, Ciência e Tecnologia de Santa Catarina pelo apoio e incentivo à pesquisa. 


\section{REFERENCIAS}

[1] IAEA/SAFRON - International Atomic Energy Agency/Safety in Radiation Oncology.

SAFRON Reports. 2019. Avaible in: <https://rpop.iaea.org/SAFRON/Report/ReportList.aspx>. Accessed in: 01.11.2019.

[2] TEIXEIRA FCS. Acidentes em Radioterapia. In: XX Congresso Brasileiro de Física Médica e Simpósio Internacional de Proteção Radiológica em Medicina. Rio de Janeiro: IPEN; 2015. [3] FAGUNDES JS, FERREIRA AF, LIMA CMA, SILVA FCA. Lições aprendidas com acidentes radiológicos nas exposições médicas em radioterapia. Braz J Rad Sci. 2018; 6(2A):01-17. https://doi.org/10.15392/bjrs.v6i2\%20-\%20A.506.

[4] REIS CT, MARTINS M, LAGUARDIA J. Patient safety as a dimension of the quality of health care - a look at the literature. Ciênc Saúde Colet. 2013 Jul; 18(7):2029-36.

[5] ZIETMAN AL, PALTA JR, STEINBERG ML, BLUMBERG AL, BURNS AR, CAGLE SW, et al. Safety is no accident, a framework for quality radiation oncology and care. ASTRO; 2012. [6] ICRP - International Commission on Radiological Protection. Prevention of Accidents to patients undergoing radiation therapy. ICRP Publication 86. Ann. ICRP. Sept 2000; 30(3):01-70. [7] OMS - Organização Mundial de Saúde. Radiotherapy risk profile - technical manual. Geneva: WHO; 2008.

[8] BERNARDES M. Aplicação de métodos de engenharia de fatores humanos para avaliação e mitigação de riscos no processo de radioterapia [dissertacão]. Itajubá: Universidade Federal de Itajubá; 2017.

[9] DEROSIER J, STALHANDSKE E, BAGIAN JP, NUDELL T. Using health care failure mode and effect analysis ${ }^{\mathrm{Tm}}$ : the VA National Center for Patient Safety's prospective risk analysis system. Jt Commun J Qual Patient Safety. 2002 May; 28(5):248-67.

[10] LI G, XU B, HE RX, ZHANG SX. Using healthcare failure mode and effect analysis to reduce intravenous chemotherapy errors in chinese hospitalized patients. Cancer Nurs. 2017; 40(2):88-93. [11] CNEN - Comissão Nacional de Energia Nuclear. Requisitos de segurança e proteção radiológica para serviços de radioterapia. Norma CNEN NN 6.10. Dez 2014. 
[12] OMS - Organização Mundial da Saúde. Bonn call for action. Joint position statement by IAEA and WHO. Geneva: WHO; 2013.

[13] DEANDREA S, TIDONE E, BELLINI A, BISANTI L, LEONARDO NG, SILVESTRI AR, et al. Implementation of Failure Mode and Effects Analysis to the specimens flow in a populationbased colorectal cancer screening programme using immunochemical faecal occult blood tests: a quality improvement project in the Milan colorectal cancer screening programme. Bmj Open Quality. 2018; 7(1):e000299.

[14] GRILLONE L, CASTRIOTTA L, BELLOMO F, SIGNOR MA, LIBERALE A, PRATILLO $\mathrm{V}$, et al. Increasing patient safety in breast cancer radiotherapy pathway: results of HFMEA performed in a large academic hospital. Eur Soc Radiol. 2017; 1-10.

[15] HABRAKEN MMP, VAN DER SCHAAF TW, LEISTIKOW IP, REIJNDERS-THIJSSEN PMJ. Prospective risk analysis of health care processes: a systematic evaluation of the use of HFMEA $^{\mathrm{TM}}$ in Dutch health care. Ergonomics. 2009 Jun; 52(7): 809-19.

[16] VLAYEN A. Evaluation of Time- and Cost-Saving Modifications of HFMEA: an experimental approach in radiotherapy. J Patient Saf. 2011 Sept; 7(3):164-7.

[17] BIAZOTTO B, TOKARSKI M. Comparison of Methods for Prioritizing Risk in Radiation Oncology. Rev Bras Fis Med. 2016; 10(1):17-21.

[18] DOROW PF, SILVA C, VARGAS F, SALVADOR C, BERTONCINI MA, NOBREGA J, ET al. Best practices for sharing knowledge in a radiotherapy service. Int J Dev Res. 2018 Sep; 8(9):22699-704.

[19] SOUZA CN, MONTI CR, SIBATA CH. Recommendations to avoid gross errors of dose in radiotherapeutic treatments. Radiol Bras. 2001 Jan-Feb; 3(1):29-37.

[20] SANTO MLR, BRUNO AC, ARRUDA FT, ARRUDA GV, BERTUCCI EC, BARBI GL, et al. Análise de riscos FMEA vs. SEVRRA em tratamentos radioterápicos com equipamentos de quilovoltagem. Braz J Rad Sci. 2019; 7(3):01-18. https://doi.org/10.15392/bjrs.v7i3.864

Este artigo foi elaborado com base na dissertação intitulada "Segurança do paciente e análise de risco na teleterapia conformacional em um centro de tratamento oncológico do sul do Brasil”, apresentada ao Programa de Pós-Graduação em Proteção Radiológica do Instituto Federal de Educação, Ciência e Tecnologia de Santa Catarina (IFSC), em 2018. 